\title{
Bases biológicas de la actividad física y salud en el entorno laboral
}

\section{Biological basis of physical activity and health in the labour environment}

Luis Felipe Varela Polit ${ }^{1}$, Víctor Manuel Hernández Toro ${ }^{2}$, Cinthia Katherine Galarza ${ }^{3}$. Galarza. Jeanneth Caroline Galarza Galarza ${ }^{4}$

\begin{abstract}
.
DOI: $\underline{\text { https://doi.org/10.33262/cienciadigital.v3i2.5.532 }}$

Objective: Explain the need to perform physical activity within the workplace as a means of reducing risk factors for health associated with the development of stress. Update information by relating the variables of physical activity and diseases in work contexts. Methodology: Apply meta-analysis to databases where the benefit of frequent physical activity during the workday is staged (in studies conducted in India, USA and Colombia) for the improvement of vital bodily functions in databases and practice guides clinic. Results: there is evidence of the relationship between regular physical activity in the work environment with simple practices such as active pauses and walking to improve vital functions, mainly increased blood flow, an improvement in nerve conduction that is evidenced by an improvement of attention capacity and improvement of work in the hippocampus, represented by a better assimilation of information as well as the creation of

\footnotetext{
${ }^{1}$ Ministerio de Salud Pública del Ecuador. Riobamba, Ecuador. luis.varela@06d05.zimbra.gob.ec

${ }^{2}$ Universidad Técnica de Ambato, Facultad de Ciencias de la Salud, Carrera de Medicina. Ambato, Ecuador.vhernandezt23@hotmail.com

${ }^{3}$ Escuela Superior Politécnica de Chimborazo, Facultad de Salud Pública, Carrera de Medicina. Riobamba, Ecuador. Cinthia.galarza@espoch.edu.ec

${ }^{4}$ Universidad Técnica de Ambato, Facultad de Ciencias Humanas y de la Educación, Carrera de Pedagogía de la actividad física. Ambato, Ecuador. jeannethcgalarzag@uta.edu.ec
} 
neuronal reverberant circuits with the improvement of long-term memory. Furthermore, apart from the organic aspect there is a considerable improvement in the maintenance of mental health as a fundamental basis of health in the new health model of the country. Conclusion: physical activity is a pillar in the maintenance and improvement of health both in personnel with previous pathologies, as well as in apparently healthy people improving their overall work performance and creating a more effective and effective work environment.

Keywords: Health, Physical activity, Biology, Mental

\section{Resumen.}

Objetivos: Actualizar información a partir de relacionar las variables de actividad física y las enfermedades en contextos laborales. Metodología: Se aplicó el enfoque cualitativo fundamentado en la herramienta del meta análisis, que consiste en comparar estudios realizados y publicados en bases de datos Pubmed, EBSCO, Cochrane, LILACS y PedDro, mediante los términos Physical, Activity, Work, Benefits y therapeutic benefits (India, USA y Colombia), en los que se explica el beneficio de la actividad física en la jornada laboral para el mejoramiento de las funciones corporales vitales y guías de práctica clínica. Resultados: Se evidencia la relación entre la actividad física regular en el entorno laboral con prácticas simples como las pausas activas y la caminata para mejorar las funciones vitales principalmente, el aumento del flujo sanguíneo, una mejora de la conducción nerviosa que se revela con el aumento de la capacidad de atención y de trabajo en el hipocampo, representándose con el incremento de la asimilación de la información, así como la creación de circuitos reverberantes neuronales lo que desencadena en la capacidad de memorización a largo plazo. Además, fuera del aspecto orgánico existe una mejoría considerable en la salud mental como base fundamental de la salud. Conclusión: La actividad física es un pilar en el mantenimiento y mejoramiento de la salud, tanto en el personal con patologías previas, así como en personas aparentemente sanas mejorando su desempeño laboral general y creando un ambiente de trabajo más eficaz y efectivo.

Palabras clave: Salud, Actividad física, Biología, Mental

\section{Introducción.}

La actividad física se conceptúa como la energía utilizada para el movimiento que le permite mantener las funciones vitales, mientras que el ejercicio, relaciona a los movimientos diseñados y planificados para gozar de buena salud (Abajo, 2018). La actividad física se ha establecido como uno de los pilares de la salud, tanto por sus beneficios como por su 
capacidad para reducir los factores de riesgo que pueden interferir con la homeostasis del cuerpo.

La ausencia de actividad física unida a los malos hábitos alimenticios son la causa de varias enfermedades y por supuesto un indicador que aumenta cada día en el mundo como es el sedentarismo (Diaz, 2011). Los períodos de descanso y actividad física con respiración, estiramientos musculares y movilizaciones articulares en diferentes segmentos corporales resultan importantes (Cáceres \& Magallanes, 2017).

De hecho, existe una lucha incesante por controlar el peso, existen varios factores contemporáneos que contribuyen a esta preocupación como los avances tecnológicos, el transporte motorizado, automatización y la máquina que ahorran trabajo física y hace que se gaste menos energía, con ese antecedente, se aplican varias estrategias para persuadir a la gente para que haga actividad física (Cintra \& Balboa, 2011).

La actividad física se relaciona directamente con la salud y por ende con la calidad de vida (Vidarte, Vélez, \& Sandoval, 2011). A pesar de que ningún medio puede detener la degeneración fisiológica del organismo, sí se puede lograr que la transición hacia la etapa de adulto mayor sea mucho menos traumática, tanto mental como físicamente, además de que permite reducir la posibilidad de padecer una enfermedad crónica degenerativa que, según la OMS (2018), registra un notable aumento en su prevalencia, tal es el caso de una enfermedad fácilmente prevenible con actividad física como la Diabetes; aumento de 1997 con 1.896 defunciones al 2011 con 4.455 defunciones.

Tabla 1. Casos de mortalidad. Fuente: INEC

Principales

\begin{tabular}{lcc}
\hline Diabetes Mellitus & 1896 & 4455 \\
Enfermedades hipertensivas & 2184 & 4381 \\
Enfermedades cerebrovasculares & 2280 & 3930 \\
Accidentes de transporte terrestre & 2007 & 3351 \\
Influenza y neumonía & 2590 & 3086
\end{tabular}

Fuente: Elaboración propia. 
Las enfermedades crónicas han logrado posicionarse dentro de las principales causas de morbilidad en el país, a pesar de que son fácilmente prevenibles; de esa manera se modificarían los factores de riesgo, como el sedentarismo con lo que se podría reducir hasta en un $80 \%$ los casos de cardiopatías y diabetes mellitus tipo 2 .

Factores biológicos de riesgo:

Modificables: sobrepeso/obesidad, hipercolesterolemia, hiperglucemia, hipertensión arterial.

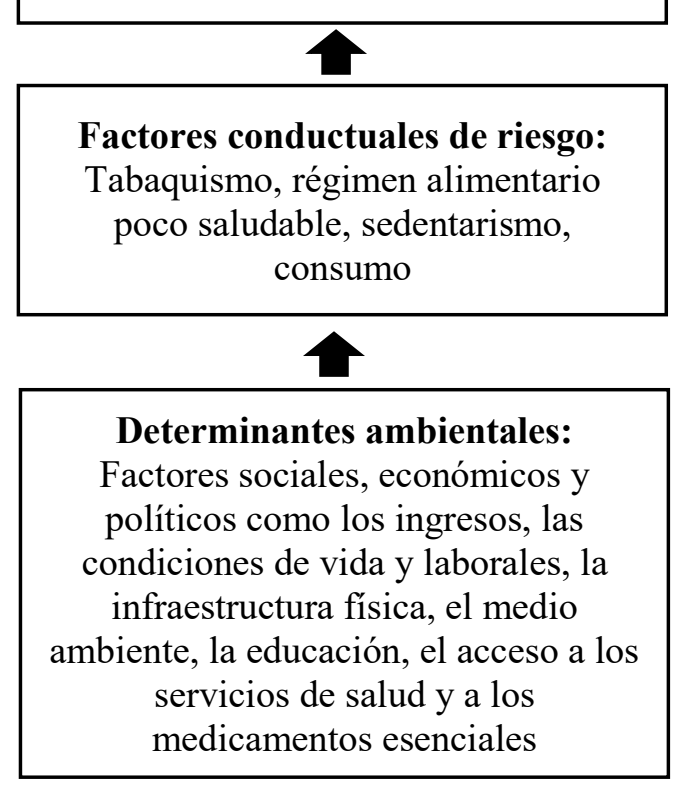

Figura 1. Determinantes clave de las enfermedades no transmisibles

Dentro de los determinantes ambientales de la salud, se ha indicado como parte de los principales problemas para el aparecimiento de las enfermedades crónicas las condiciones de vida laboral, dentro de las cuales se indica que la actividad física dentro del entorno laboral reduce los factores de riesgo, y disminuir alrededor de 1,6 millones (OMS, 2018) de muertes anuales se asocian únicamente a la actividad física insuficiente.

Un concepto que desde hace bastante tiempo se implementa en el ámbito laboral es incentivar la productividad de sus empleados mediante actividades recreativas y pausas para disminuir el estrés laboral mediante la realización de ejercicios (Ashe, 2012).

Entre los lugares donde se ha puesto en práctica esta modalidad con mayor énfasis son las industrias asiáticas, donde es frecuente observar al personal realizando una rutina corta de aproximadamente 10 minutos de ejercicios de calistenia (Ocupacional, 2002), a partir de ello, en varios países del mundo, donde se puede destacar a Suecia plantean una variante diferente, ya que decidieron convertir el ejercicio físico en una obligación laboral, cada viernes los empleados abandonan la sede laboral para dirigirse a un centro de entrenamiento deportivo cercano, donde deben cumplir una hora semanal de yoga, aeróbico u otra modalidad. 
Además, detrás de ello, hay otra razón. Un estudio de la Universidad de Estocolmo (2011) demostró que practicar una actividad física moderada durante la jornada laboral otorgaba la ventaja de poder realizar el trabajo concentrado y reducir riesgo de desarrollar enfermedades crónicas (para el empleado) y de conseguir una reducción de hasta el $22 \%$ del absentismo laboral.

Por ejemplo, las actividades prolongadas que demanda un puesto de trabajo, la fatiga y la falta de recuperación son condiciones que expresan una falta de equilibrio entre el esfuerzo físico y la capacidad que tiene el trabajador para ejecutarlo; por ello, se hace imprescindible el uso de estrategias en prevención del riesgo laboral, fundamentadas -entre otras- en la actividad física, para prevenir y tratar las enfermedades derivadas de la ocupación (Almagro. 2014).

Entre los factores que contribuyen a afectar la salud de la población trabajadora se encuentran los movimientos repetitivos, las posturas estáticas, las posturas incómodas y el sobre esfuerzo, que puede rebasar los límites físicos y de carga del individuo; en otras palabras, la salud puede verse afectada por aspectos multicausales (Martínez, 2017).

\section{Metodología.}

El presente meta análisis pretende actualizar información respecto de la ejecución o falta de actividad física con el desarrollo que patologías dentro del entorno laboral; con esos datos crear gráficos representativos tipo Forrest Plot para demostrar el beneficio, ya sea para el grupo de estudio o el grupo control y de esta manera incentivar la realización de actividad física de manera regular en el ámbito laboral.

Criterios de selección

Se realizó una búsqueda de artículos científicos en donde se exprese la relación entre la poca actividad física y el desarrollo de enfermedades en el ámbito laboral. La búsqueda se realizó en las bases de datos Pubmed, EBSCO, Cochrane, LILACS y PedDro, mediante los términos Physical, Activity, Work, Benefits y therapeutic benefits. Los operadores utilizados fueron "AND” y las búsquedas fueron dirigidas desde un enfoque general hasta el más específico.

Debido a la naturaleza de la revisión, y desde una búsqueda inicial que incluyó "todos los campos", se depuró la información encontrada a 32 artículos; cada uno de ellos, con estudios que relacionaban la actividad física leve o nula y su impacto en el desarrollo de enfermedades de tipo crónicas no transmisibles en contextos laborales. Los criterios de exclusión tomados en cuenta fueron la aparición de un artículo en varias bases de datos y la duplicidad de resultados por búsqueda, eliminando aquellos no referentes al objeto de estudio o artículos desactualizados. 


\section{Resultados}

Salud mental

La depresión es una enfermedad común en el envejecimiento de la población y la probabilidad de padecer esta enfermedad psicológica es de dos a tres veces mayor en pacientes con enfermedades crónicas (Barbosa, 2018; Wilner, 2014). Muchos estudios transversales han demostrado que los pacientes deprimidos son más sedentarios y por ende tienen mayor riesgo para el desarrollo de patologías crónicas (Weyerer, 2014).

En los últimos tiempos, se ha estudiado la aplicación de actividad física como tratamiento para la prevención de la depresión (Barbosa, 2018). En este sentido, Países como, Reino Unido, Estados Unidos y Australia, han desarrollado pautas de actividad física relacionadas con la salud, en las cuales se recomienda al menos 30 minutos de actividad moderada en 2 tiempos durante la jornada laboral, todos o casi todos los días de la semana (Bonet, 2014)

Estas directrices fueron desarrolladas para mejorar la salud de la población y para reducir el riesgo de padecer depresión y en pacientes ya diagnosticados, con o sin tratamiento, reducir el riesgo de desarrollar patológicas crónicas.

\section{Sistema Nervioso}

La práctica de la actividad física está relacionada con la mejora de la conducción neuronal en el cerebro, diencéfalo, tronco encefálico y cerebelo. La actividad física potencializa a nivel cerebral la angiogénesis, las propiedades electrofisiológicas y el aumento de los factores de crecimiento cerebrales, la plasticidad neuronal relacionada con la formación de circuitos reverberantes para lograr utilizar información a largo plazo, además de creación de nuevos circuitos neuronales para la adquisición de nuevas destrezas.

La práctica de la actividad física en modelos animales sugiere un aumento en la cantidad de astrocitos en las estructuras motoras del cerebelo.

Núcleo del rafe dorsal

Contiene neuronas serotoninérgicas las cuales proyectan serotonina a varias regiones del cerebro, las cuales actúan en el control del estado de ánimo y el comportamiento sobre el cual se demostró según Morgan (2015) que 6 semanas de actividad física en bicicleta estática es efectiva en la reducción incontrolable del estrés y el déficit de atención.

\section{Locus Ceruleo}

Actúan en la regulación de la atención y la vigilancia de los niveles de estrés misma que es controlada mediante señalizaciones vía norepinefrina en conjunto con la galanina (péptido regulador) que atenúan la hiperexcitabilidad neuronal y puede estar relacionada con el 
control noraderenérgico del estrés por lo que la producción de galanina podría contribuir a la respuesta al estrés agudo.

Adaptación inducida por el ejercicio sobre los ganglios basales

De todos los núcleos basales tienen actividad en el control del tono muscular y el movimiento por su acción sobre la corteza motora y las placas neuromusculares, especialmente en putamen) el ejercicio intenso podría tener acción en estos núcleos bloqueando las vías de la ERK $1 \frac{1}{2}$ y CREB que se asocian a deficiencias en la memoria implícita (Morgan, 2015).

Además el ejercicio moderado incrementa la liberación estrial de la Sintetasa de Óxido Nítrico importante en la plasticidad sináptica y en caso de deficiencia de la misma se puede relacionar al desarrollo de enfermedades degenerativas (Morgan, 2015).

Adaptación a la respuesta inmune

Evidencia científica determina que la práctica regular de la actividad física permea el incremento y el mantenimiento de la salud (sistemas de neuroprotección), la disminución de la frecuencia-intensidad de malestares y la incidencia de nuevas enfermedades a través de la activación de genes antienvejecimiento y el aumento de la función inmune.

Tejido adiposo

Al realizar una actividad física se queman calorías, esto lleva al organismo al consumo de lípidos del tejido adiposo como fuente de energía que acelera la pérdida de grasa corporal concomitante a la activación de la placa motora, el refortalecimiento de tendones y ligamentos al hueso; y la disminución de niveles de colesterol y triglicéridos, el aumento de la densidad mineral, la mejora la consolidación de las estructuras óseas, el incremento en la modulación de la temperatura.

Aparato Cardiovascular

Otro meta-análisis, analizó los efectos de la actividad física moderada frente a la inactividad en el ámbito laboral y en el tiempo libre, en relación con los accidentes cerebrovasculares, y mostró un efecto protector para los individuos que realizaban actividad física en el trabajo ( $\mathrm{RR}=0,64 ; 95 \%$ IC; $0,48-0,87)$ así como también para los que realizaban actividad física en el tiempo libre ( $R R=0,85,95 \%$ IC; 0,78-0,93) (López 2016). De los estudios incluidos en ese meta-análisis, dos de ellos fueron seleccionados para esta tesis (Li 2012), García (2015), se observó que, en estudios de cohortes, los individuos que realizaban actividad física con intensidades altas obtenían un efecto protector $(\mathrm{RR}=0.75 ; 0.69-0.82 ; 95 \% \mathrm{IC})$. 


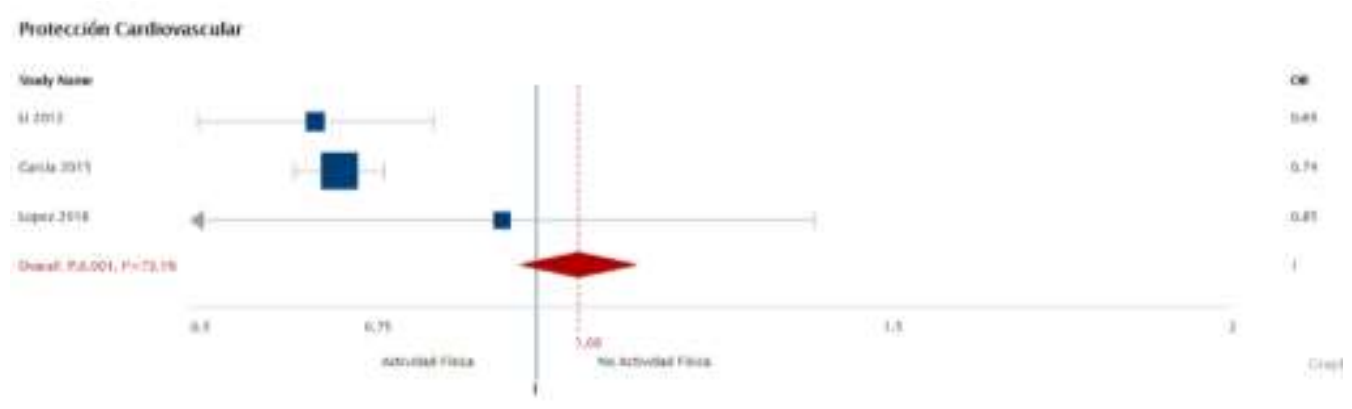

Figura 2. Protección Cardiovascular

Elaborado por: Equipo investigador

Cognición y conducta

Se hace evidente la relación de la actividad física con el incremento-mantenimiento de niveles de salud mental, el aumento de niveles de espiritualidad y la proyección funcional de expectativas futuras del mismo modo, la práctica regular de esta actividad mejora la función cognitiva, representada en habilidades de aprendizaje y/o funcionamiento intelectual, de percepción, de atención, de concentración, de memoria y otras funciones mentales ejecutivas no especificadas como demuestran De Giorgo (2018).

Gracias a la práctica de la actividad física se reflejan cambios en el comportamiento como, por ejemplo, la disminución en la incidencia de una conducta antisocial, hostil, fóbica, psicótica, en su estado de ánimo, sensación de bienestar, autodeterminación, confianza, autocontrol y asertividad; además de minimizar el abuso de alcohol, los eventos de ira, confusión, ansiedad y depresión.

Promoción de la salud

Promoción de la salud y prevención de la discapacidad

La práctica de la actividad física está relacionada con la disminución de la mortalidad y morbilidad por enfermedad, el retraso en el declive funcional y la reducción del riesgo de padecer enfermedades crónicas; en estos términos, vale la pena considerar que su práctica constante ayuda a prevenir el sobrepeso y la obesidad, (Cordero, 2014) disminuye las actitudes sedentarias e incluso previenen su aparición con el avance de los años (Landinez, 2012). Algunas de las aplicaciones reportadas por la literatura van dirigidas al manejo del síndrome metabólico diabetes mellitus, hipertensión arterial, dislipidemias,artrosis (Márquez, 2014).

Sistema musculo esquelético 
Un estudio transversal reciente, realizado en más de 600 trabajadores con trastornos músculo esqueléticos relacionados con las extremidades superiores, demostró la prevalencia de dolor del $60 \%$ de los casos; para el codo del $40 \%$ y en las manos de un $52 \%$. Según Moreria CR (2016) mantenerse activo durante los momentos de ocio se asocia de una disminución del riesgo de sufrir patologías ocupacionales en el hemicuerpo superior.

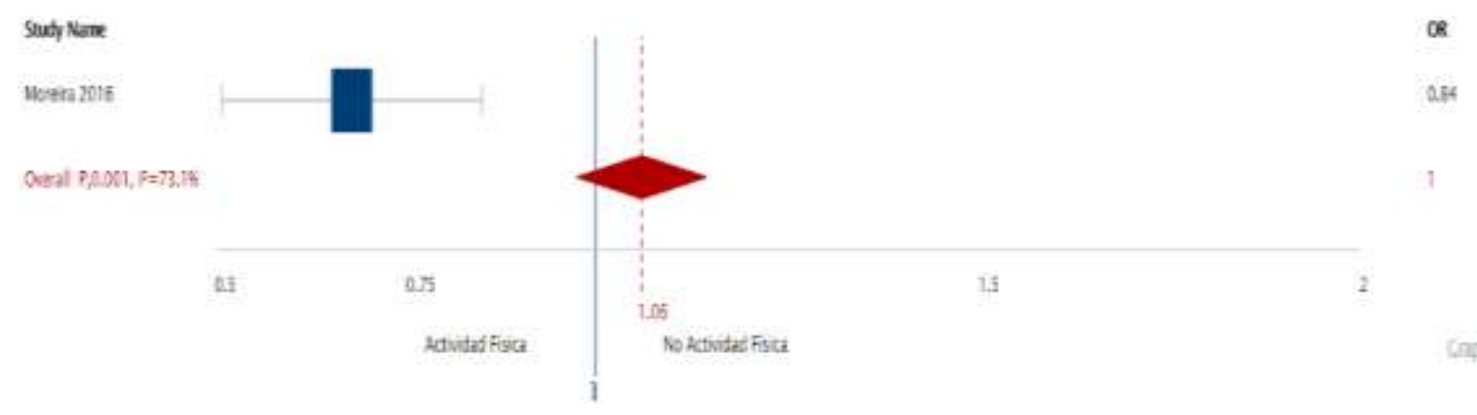

Figura 3. Actividad Física

Elaborado por: Equipo investigador

Según diversos estudios, iniciativas efectivas en los lugares de trabajo deberían incluir programas de nutrición y programas que potencien la actividad física laboral en etapas para reducir el dolor musculo esquelético relacionado con la posición fija prolongada y la poca movilidad dando al individuo una mejora funcional, resistencia física, fuerza muscular y reducción del dolor localizado al aumentar la densidad ósea y mejorar la postura (Rasmussen., 2017; Wolfenden (2018) Tunceli, Li, \& Williams, 2016).

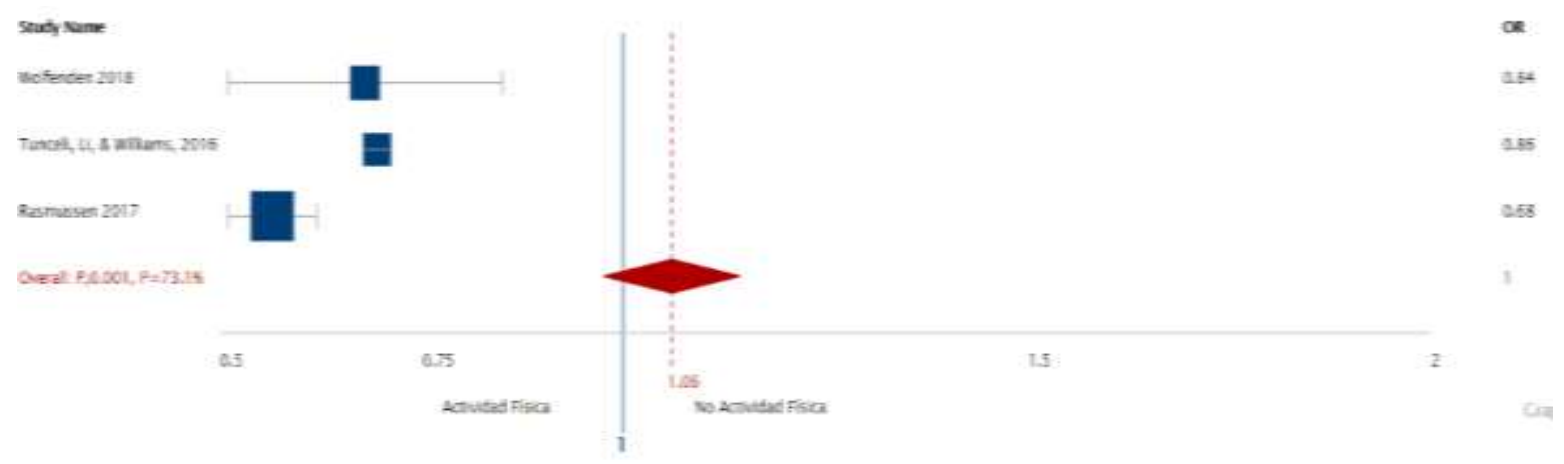

Figura 4. No Actividad Física

Elaborado por: Equipo investigador 


\section{Conclusiones.}

La actividad física además de mejorar el estado general del organismo tiene la capacidad de reducir la incidencia de las enfermedades crónico-degenerativas hasta en un $80 \%$ y así reducir el riesgo de discapacidades y de incrementar la mortalidad en el país.

De acuerdo a los estudios citados anteriormente y al tamaño del efecto medio obtenido en el estudio meta-analítico sobre los niveles de actividad y su beneficio a la salud, se verifica que los resultados obtenidos son congruentes y similares. En función de ello, se puede concluir que la práctica de actividad física en forma moderada ofrece un efecto protector sobre los individuos que la realizan, disminuyendo en un $22 \%$ el riesgo de estos a padecer enfermedades cardiovasculares.

Parte importante de la salud actualmente es la salud mental, la actividad física puede reducir el riesgo de depresión tanto en el ámbito laboral como en la vida diaria aumentando la eficiencia del personal y eliminando el riesgo de absentismo laboral, esto, con tan poco esfuerzo como 30 minutos al día dividido en 2 momentos durante la jornada.

\section{Referencias bibliográficas.}

Almagro Valverde, S.; Dueñas Guzmán, M.A. y Tercedor Sánchez, P. (2014). Actividad física y depresión: revisiónsistemática / Physical activity and depression: a systematic review. Revista Internacional de Medicina y Ciencias de la Actividad Física y el Deporte vol. 14 (54) pp. 377- 392.

Ashe, M. C. (2012). Physical activity and workplace sedentary behaviour. Physiotherapy Canada, 64, 1-3. doi:10.3138/ptc.64.1.ge1

Christensen, J., Faber, A., Ekner, D., Overgaard, K., Holtermann, A., Sogaard, K. (2011). Diet, physical exercise and cognitive behavioral training as a combined workplace based intervention to reduce body weight and increase physical capacity in health care workers-A randomized controlled trial. BMC Public Health, 11(1), Article 671. doi:10.1186/1471-2458-11-671

Cordero A, Masiá MD, Galve DE (2014). Ejercicio físico y salud. Rev Española Cardiol.; 67(9): 748-53.

Sarsak HI (2018) The Occupational Therapy Process in Psychosocial Disorders: An Overview. Jr Neurology, Psy and Brain Res: JNPBR-113.

De Giorgio A., Kuvačić G., Milić M., Padulo J., (2018) The Brain and Movement: How Physical Activity Affects the Brain., Monten. J. Sports Sci. Med. 7 (2018) 
del Val MP. (2013) Relación entre la actividad física y el rendimiento académico. Valladolid: Universidad de Valladolid, Facultad de Educación y Trabajo Social;.

Feter N., Freitas M.P., Gonzales N.G. (2017) Effects of physical exercise on myelin sheath regeneration: A systematic review and meta-analysis., Science \& Sports 33(1):8-21 - August 2017

García, C. (2015). Eficacia de la Actividad Física en la reducción del riesgo de enfermedades cardiovasculares, Un Meta-análisis. Tesis de posgrado. Universidad Nacional de La Plata. Facultad de Humanidades y Ciencias de la Educación.

Holtermann, A., Mortensen, O.S., Burr, H., Søgaard, K., Gyntelberg, F., \& Suadicani, P. (2009). The interplay between physical activity at work and during leisure time - risk of ischemic heart disease and all-cause mortality in middle-aged Caucasian men. Scand. J. Work Environ Health, 35 (6), 466-474. doi:10.5271/sjweh.1357.

HUTCHINSON A., WILSON C., (2011) Improving nutrition and physical activity in the workplace: a meta-analysis of intervention studies. Health Promotion International, Vol. 27 No. 2

Landinez PNE, Contreras VK, Castro VA. (2012) Proceso de envejecimiento, ejercicio y fisioterapia. Rev Cubana Salud Pública.; 38(4): 562-80.

Li, A., Siegrist, J. (2012). Physical Activity and Risk of Cardiovascular Disease-A MetaAnalysis of Prospective Cohort Studies. Int. J. Environ. Res. Public Health, 9 (2), 391-407. doi:10.3390/ijerph9020391.

Löllgen H., 1, Böckenhoff A., Knapp G., (2009) Physical Activity and All-cause Mortality: An Updated Meta-analysis with Diff erent Intensity Categories., Int J Sports Med 2009; 30: $213-224$

López SLM, Pérez HAJ, Sisa ÁMA, Téllez LLN.( 2016) Factores de riesgo cardiovascular en funcionarios de una institución gubernamental en Tunja, Colombia. Rev Cuidarte.; 7(2): 1279-87.

Márquez AJJ, Márquez AWH.( 2014) Artrosis y actividad física. Rev Cubana Ortop Traumatol.; 28(1): 83-100.

Martínez GJA, García IS, Castellanos SVO. (2015) Ergonomía, expresiones de movimiento incidentes en la salud y la ocupación de trabajadores de la industria metalmecánica. Rev Terapia Ocupacional Galicia.; (22): 23.

Miranda, H., Viikari-Juntura, E., Martikainen, R., Takala, E. P., Riihimäki, H. (2014). Physical exercise and musculoskeletal pain among forest industry workers. 
Scandinavian Journal of Medicine \& Science in Sports, 11, 239-246. doi:10.1034/j.16000838.2001.110408.x

Moreria I., Teixeria P., (2016) The Effects of Workplace Physical Activity Programs on Musculoskeletal Pain: A Systematic Review and Meta-Analysis. Sage Journals Vol 64, Issue 5,2016

Morgan,J. Corrigan,F. Baune B (2015) Effects of physical exercise on central nervous system functions: a review of brain region specific adaptations J Mol Psychiatry. 3: 3.

Organización Mundial de la Salud (2018). Enfermedades no transmisibles

Organizacion Panamericana de la Salud (2011). Las enfermedades no transmisibles en la Región de las Américas: Todos los sectores de la sociedad pueden ayudar a resolver el problema

Barbosa, S., Urrea, A. (2018). Influencia del deporte y la actividad física en el estado de salud físico y mental: una revisión bibliográfica. Revista Katharsis, N 25, enero-junio 2018, pp.141-159,

Peña, M., Acuña, C., Montoya R, (2014). La nueva situación epidemiológica de Ecuador, Situación de las enfermedades crónicas no transmisibles. Revista Informativa., 32, 14-21

Rasmussen NCH, Lindberg NK, Ravn MH, Jorgensen MB, Sogaard K, Holtermann A. (2017) Processes, barriers and facilitators to implementation of a participatory ergonomics program among eldercare workers. Appl Ergon.; 58(1): 491-9.

Newman, t. (2015). Medical news today, repetitive strain injury: treatments and prevention. Directions, 1, 26-27

Riquelme UD, Sepúlveda GC, Muñoz MM, Valenzuela HM.( 2013) Ejercicio físico y su influencia en los procesos cognitivos. Rev Motricidad Persona.; 13(1): 69-74.

Rodríguez ML, Díaz CR. (2012) Beneficios del ejercicio físico terapéutico en pacientes con secuelas por enfermedad cerebrovascular. Rev Cubana Med Gen Integr.; 51(3): 258-66.

Romero C., (2015) Meta-análisis del efecto de la actividad física en el desarrollo de la resiliencia Retos, número 28, 2015 ( $2^{\circ}$ semestre)

Sundstrup E, Jakobsen MD, Brandt MJK, Aagaard P, Andersen L. (2016)Strength training improves fatigue resistance and self-rated health in workers with chronic pain: A randomized controlled trial. BioMed Res Int. 2016; 2016: 2-10.

Tunceli, K., Li, K., Williams, L. K. (2016). Long-term effects of obesity on employment and work limitations among U.S. adults, 1986 to 1999. Obesity, 14, 1637-1646. 
Wolfenden L, Goldman S, Stacey FG, Grady A, Kingsland M, Williams C, Wiggers J, Milat A, Rissel C, Bauman A, Farrell MM, Légaré F, Ben Charif A, Zomahoun H, Hodder RK, Jones J, Booth D, Parmenter B, Regan T, Yoong S., (2018) Improving the implementation of health-promoting policies and practices in workplaces, Cochrane 14 November

Wolfenden L, Goldman S, Stacey FG, Grady A, Kingsland M, Williams CM, Wiggers J, Milat A, Rissel C, Bauman A, Farrell MM, Légaré F, Ben Charif A, Zomahoun HTV, Hodder RK, Jones J, Booth D, Parmenter B, Regan T, Yoong SL.( 2018) Strategies to improve the implementation of workplace-based policies or practices targeting tobacco, alcohol, diet, physical activity and obesity. Cochrane Database of Systematic Reviews, Issue 11. Art

Wilner, N.J., Tone, E.B. (2014). Physical activity and stress resilience: Considering those AtRisk for developing mental health problems, Mental Health and Physical Activity, 8, 1-7

Bonet J., Parrado E., Capdevila L., (2017), Efectos agudos del ejercicio físico sobre el estado de ánimo, 17, 85-100

\section{PARA CITAR EL ARTÍCULO INDEXADO.}

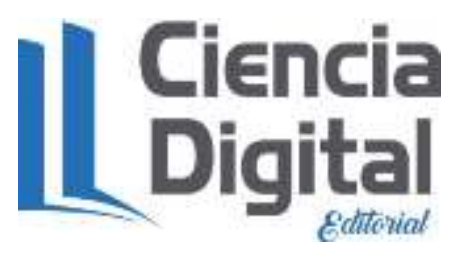

Varela Polit, L., Hernández Toro, V., Galarza Galarza, C., \& Galarza Galarza, J. (2019). Bases biológicas de la actividad física y salud en el entorno laboral. Ciencia Digital, 3(2.5), 77-89. https://doi.org/10.33262/cienciadigital.v3i2.5.532

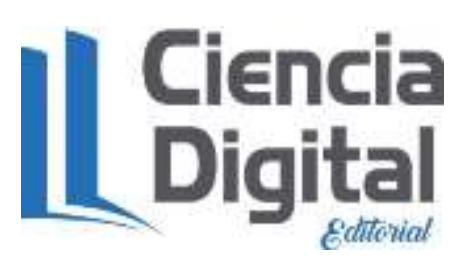

El artículo que se publica es de exclusiva responsabilidad de los autores y no necesariamente reflejan el pensamiento de la Revista Ciencia Digital.

El artículo queda en propiedad de la revista y, por tanto, su publicación parcial y/o total en otro medio tiene que ser autorizado por el director de la Revista Ciencia Digital.
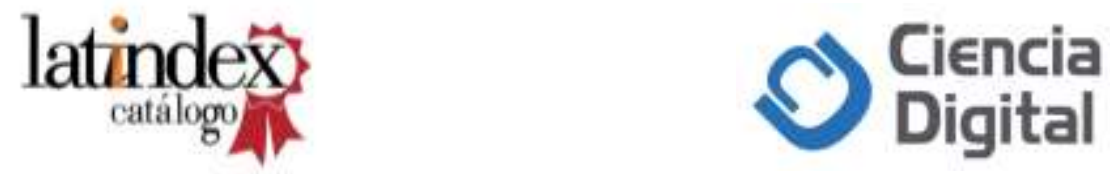